\title{
Dynamics of 3 - Links Articulated Robotic Manipulator: A Computational Model
}

\author{
Chukwuemeka C. Obasi, Ikharo A. Braimoh, Alphaeus Odaba, Leonard Iyase Ogbewey, Bambe \\ A. Oluyomi
}

\begin{abstract}
Dynamic computation include the process of determining the forces and energies that would cause a manipulator to move certain distance at a given angle. The complex nature of available materials has made this process difficult. The dynamics equation for a 3-links robotic manipulator was designed using the Lagrange archetypal. The result shows that the energies (including Potential and Kinetic Energy) as well as the torques required to cause motion at each joint can be computed separately. The torque equations represents the dynamic models required.
\end{abstract}

Keywords: Dynamic equation; Robotic manipulator; force; energy; Newton-Euler; Lagrange.

\section{INTRODUCTION}

Machines that possess the ability to automatically carry out tasks with little or no human aid is referred to as Robots [8]. Robotics is therefore the sum of all actions that are directed towards the design, development and study of robots. Robotics is a complex system that requires the efforts of multi-discipline to develop, which is necessitated by the involvement of different parts requiring various forms of controls energies by actuators providing forces or torque [1, 2]. The development of Robotic system involves multiple steps of complex computational estimation for necessary torque to enable motion for the manipulator. The manipulator being an electronically controlled mechanism with linkages, otherwise known as the robot arm [9]. One of these complexity involves the estimation of the dynamics of the robotic system, which implies the computation of the forces or torque that would act at the various joint and hence creating motion on the entire arm $[1,2]$. According to

Revised Manuscript Received on February 05, 2020.

* Correspondence Author

Chukwuemek C. Obasi*, Department of Computer Engineering, Edo University Iyamho, Edo State Nigeria.

Email: Obasi.c_chukwuemeka@yahoo.com.

Ikharo A. Braimoh, Department of Computer Engineering, Edo University Iyamho, Edo State, Nigeria.

Email: ikharo.braimoh@edouniversity.edu.ng

Alphaeus Odaba, Electrical Electronics Engineering Department, Air Force Institute of Technology, Kaduna, Nigeria. (email: alpheous17@yahoo.com)

Leonard Iyase Ogbewey, Computer Engineering, Federal Polytechnic, Offa, Kwara State, Nigeria (email: Leonard_Ogbewey@yahoo.com)

Bambe Adeduntan Oluyomi, Computer Engineering, Federal Polytechnic, Offa, Kwara State, Nigeria (email: oluwatooni80@gmail.com)

(C) The Authors. Published by Blue Eyes Intelligence Engineering and Sciences Publication (BEIESP). This is an open access article under the CC BY-NC-ND license (http://creativecommons.org/licenses/by-nc-nd/4.0/)
Featherstone in [3], robot dynamics is a concept that explains the relationship between the force acting on the actuator of a robot manipulator that produces equivalent acceleration. Featherstone [3] went further to classify robot dynamics into two main category, including Forward Dynamic, which is the computation of acceleration when the force is known, and Inverse Dynamics, which is the computation of the force when the acceleration is known.

The development of robot dynamics has attracted numerous research in years gone by, of which Lewis in [2] was one, where the Lagrange-Euler Dynamics of motion was used to represent the general form of robot dynamics. Newton-Euler and Lagrange approaches for the formulation of robot manipulator dynamic have been presented by Craig in [5] and Asada in [3]. Both inferred that Newton-Euler approach is force based, while Lagrange approach is energy based. However, Asada claims that the former is known with the issue of force constraint, which the later provided the solution. Moreover, a generalized form of robot manipulator dynamics was presented in both cases. A review of computational algorithm of the robotic dynamics for tree-structured mechanism was presented by Featherstone in [7], where the equations were generalized still. The dynamic computation of 2-links planar manipulator was presented by Jazar in [6] and by Lewis in [2], where both used the Lagrange approach for the computation. The computational analysis of 3 - Link Articulate Robot Manipulator was presents by Obasi et al. in [10].

This paper intends to formulate the dynamics of a 3-linkes articulated robotic manipulator using the Lagrange formulation approaches. This is directed towards providing necessary information for a guide in the study of robotic manipulators in a more simplified way. This is a follow up on the work presented in [10] by Obasi et al.

\section{METHODOLOGY}

The equation of the Lagrange formulation method is shown in Eq. 1 below:

$$
\frac{d}{d t} \frac{\partial L}{\partial \dot{q}}-\frac{\partial L}{\partial q}=\tau
$$

eq. 1

Where $q$ is n-vector of generalized coordinates $q_{i}, \tau$ is an $\mathrm{n}$-vector of the generalized forces $\tau_{i}$, and $\mathrm{L}$ the Lagrangian is the difference between the kinetic and potential energies $(\mathrm{L}=$ $\mathrm{KE}-\mathrm{PE})$. 


$$
K E=\sum_{i=1}^{n} K E(\theta), P E=\sum_{i=1}^{n} P E(\theta)
$$

Where PE and KE are the total potential and kinetic energies at $l_{0}, l_{1}$, and $l_{2}$ respectively. Hence, sum of all the energies at the joints.

$\sum_{i=1}^{n} K E(\theta)=K E_{l_{0}}+K E_{l_{1}}+K E_{l_{2}}$ and $\sum_{i=1}^{n} P E(\theta)=$ $P E_{l_{0}}+P E_{l_{1}}+P E_{l_{2}}$

Now calculating the energies at each joint given in free body diagram shown in figure 1 below:

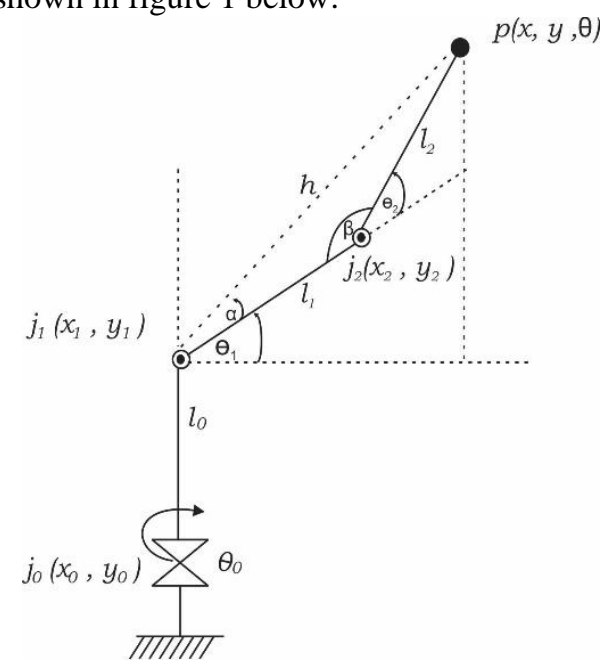

Figure 1: Free body diagram of the three links manipulator [10]

The base of the robot performs only rotational motion. Hence the energies at the base are:

$$
K E_{\text {rot }}=\frac{1}{2} m r^{2} \dot{\theta}_{0}^{2} \text { or } K E_{\text {rot }}=\frac{1}{2} I_{0} \dot{\theta}_{0}{ }^{2}
$$

Where I = inertia

Here, $\mathrm{PE}=0$ (rotational motion)

Linear energies at the links:

$$
\begin{array}{cc}
K E_{\text {linear }}=\frac{1}{2} m_{i} v_{i}^{2} & \text { Eq. } 3 \\
v_{i}{ }^{2}=\left(\dot{x}_{1}{ }^{2}+\dot{y}_{1}{ }^{2}\right) & \text { Eq. } 4 \\
P E_{\text {linear }}=m_{i} g h_{i} & \text { Eq. } 5
\end{array}
$$

At Joint J0

$$
K E_{l_{0}}=\frac{1}{2} I_{0} \dot{\theta}_{0}^{2}, P E=0
$$

\section{At Joint J1}

$$
x_{1}=l_{1} \cos \theta_{1} \text { and } y_{1}=l_{1} \sin \theta_{1}
$$

Eq. 6

$$
\dot{x}_{1}=-l_{1} \dot{\theta_{1}} \sin \theta_{1} \text { and } \dot{y}_{1}=l_{1} \dot{\theta}_{1} \cos \theta_{1}
$$

Putting Eq. 6 and Eq. 7 into Eq. 4,

$$
v_{1}^{2}=\left(-l_{1} \dot{\theta}_{1} \sin \theta_{1}\right)^{2}+\left(l_{1} \dot{\theta}_{1} \cos \theta_{1}\right)^{2}
$$

Computing the kinetic energy at this joint, Eq. 8 was put into Eq. 3 as follows:

$K E_{1}=\frac{1}{2} m_{1}\left(\left(-l_{1} \dot{\theta}_{1} \sin \theta_{1}\right)^{2}+\left(l_{1} \dot{\theta}_{1} \cos \theta_{1}\right)^{2}\right)$

Eq. 9

Also,

$$
P E_{1}=m_{1} g l_{1} \sin \theta_{1}
$$

Eq. 10

\section{At Joint $\mathbf{J}_{2}$}

$$
\begin{aligned}
& v_{2}{ }^{2}=\left(-l_{1} \dot{\theta}_{1} \sin \theta_{1}-l_{2} \dot{\theta}_{2} \sin \theta_{2}\right)^{2}+\left(l_{1} \dot{\theta}_{1} \cos \theta_{1}+\right. \\
& \left.l_{2} \dot{\theta}_{2} \cos \theta_{2}\right)^{2} \text { Eq. } 11 \\
& K E_{2}=\frac{1}{2} m_{2}\left(\left(-l_{1} \dot{\theta}_{1} \sin \theta_{1}-l_{2} \dot{\theta}_{2} \sin \theta_{2}\right)^{2}+\right. \\
& \left.\left(l_{1} \dot{\theta}_{1} \cos \theta_{1}+l_{2} \dot{\theta}_{2} \cos \theta_{2}\right)^{2}\right) \quad \text { Eq. } 12 \\
& P E_{2}=m_{2} g\left(l_{1} \sin \theta_{1}+l_{2} \sin \theta_{2}\right)
\end{aligned}
$$

Now the sum of kinetic energy is

$$
\begin{aligned}
\sum_{i=0,1,2}^{2} K E(\theta)= & \frac{1}{2} I_{0} \dot{\theta}_{0}^{2} \\
& +\frac{1}{2} m_{1}\left(\left(-l_{1} \dot{\theta}_{1} \sin \theta_{1}\right)^{2}\right. \\
& \left.+\left(l_{1} \dot{\theta}_{1} \cos \theta_{1}\right)^{2}\right) \\
& +\frac{1}{2} m_{2}\left(\left(-l_{1} \dot{\theta}_{1} \sin \theta_{1}-l_{2} \dot{\theta}_{2} \sin \theta_{2}\right)^{2}\right. \\
& \left.+\left(l_{1} \dot{\theta}_{1} \cos \theta_{1}+l_{2} \dot{\theta}_{2} \cos \theta_{2}\right)^{2}\right)
\end{aligned}
$$

$$
\begin{aligned}
= & \frac{1}{2} I_{0} \dot{\theta}_{0}+\frac{1}{2} m_{1}\left(l_{1}^{2} \dot{\theta}_{1}{ }^{2} \sin ^{2} \theta_{1}+l_{1}{ }^{2} \dot{\theta}_{1}{ }^{2} \cos ^{2} \theta_{1}\right)+ \\
& \frac{1}{2} m_{2}\left(\left[l_{1}{ }^{2} \dot{\theta}_{1}{ }^{2} \sin ^{2} \theta_{1}+2 l_{1} l_{2} \dot{\theta}_{1} \dot{\theta}_{2} \sin \theta_{1} \sin \theta_{2}+\right.\right. \\
& \left.l_{2}{ }^{2} \dot{\theta}_{2}{ }^{2} \sin ^{2} \theta_{2}\right]+ \\
& {\left[l_{1}{ }^{2} \dot{\theta}_{1}{ }^{2} \cos ^{2} \theta_{1}+\right.}
\end{aligned}
$$




$$
\begin{gathered}
\left.\left.2 l_{1} l_{2} \dot{\theta}_{1} \dot{\theta}_{2} \cos \theta_{1} \cos \theta_{2}+l_{2}{ }^{2} \dot{\theta}_{2}{ }^{2} \cos ^{2} \theta_{2}\right]\right) \\
= \\
\frac{1}{2} I_{0} \dot{\theta}_{0}+\frac{1}{2} m_{1} l_{1}{ }^{2} \dot{\theta}_{1}{ }^{2}\left(\sin ^{2} \theta_{1}+\cos ^{2} \theta_{1}\right)+ \\
\frac{1}{2} m_{2}\left(\left[l_{1}{ }^{2} \dot{\theta}_{1}{ }^{2}\left(\sin ^{2} \theta_{1}+\cos ^{2} \theta_{1}\right)+l_{2}{ }^{2} \dot{\theta}_{2}{ }^{2}\left(\sin ^{2} \theta_{2}+\right.\right.\right. \\
\left.\left.\left.\cos ^{2} \theta_{2}\right)\right]+\left[2 l_{1} l_{2} \dot{\theta}_{1} \dot{\theta}_{2}\left(\cos \theta_{1} \cos \theta_{2}+\sin \theta_{1} \sin \theta_{2}\right)\right]\right)
\end{gathered}
$$

Eq. 14

But

$$
1=\cos ^{2} \alpha+\sin ^{2} \beta
$$

$$
\cos (\alpha-\beta)=\cos (\alpha) \cos (\beta)+\sin (\alpha) \sin (\beta) \quad \text { Eq. } 16
$$

Simplifying Eq. 14 with Eq. 15 and Eq. 16,

$$
\begin{gathered}
K E=\frac{1}{2} I_{0} \dot{\theta}_{0}+\frac{1}{2} m_{1} l_{1}{ }^{2} \dot{\theta}_{1}{ }^{2}+\frac{1}{2} m_{2} l_{1}{ }^{2} \dot{\theta}_{1}{ }^{2}+\frac{1}{2} m_{2} l_{2}{ }^{2} \dot{\theta}_{2}{ }^{2} \\
+\frac{1}{2} m_{2} 2 l_{1} l_{2} \dot{\theta}_{1} \dot{\theta}_{2}\left(\cos \theta_{1}-\theta_{2}\right)
\end{gathered}
$$

$$
\begin{array}{r}
K E=\frac{1}{2} I_{0} \dot{\theta}_{0}+\frac{1}{2}\left(m_{1}+m_{2}\right) l_{1}{ }^{2} \dot{\theta}_{1}{ }^{2}+\frac{1}{2} m_{2} l_{2}{ }^{2} \dot{\theta}_{2}{ }^{2}+ \\
m_{2} l_{1} l_{2} \dot{\theta}_{1} \dot{\theta}_{2}\left(\cos \theta_{1}-\theta_{2}\right) \text { Eq. } 17
\end{array}
$$

Potential energy was calculated thus:

$$
\begin{array}{r}
\sum_{i=0,1,2}^{2} P E(\theta)=m_{1} g l_{1} \sin \theta_{1}+m_{1} g\left(l_{1} \sin \theta_{1}+\right. \\
\left.l_{2} \sin \theta_{2}\right) \text { Eq. } 18
\end{array}
$$

Using the equation, $L=P E+K E$, Hence,

$$
\begin{aligned}
\therefore L=\frac{1}{2} I_{0} \dot{\theta}_{0}+ & \frac{1}{2}\left(m_{1}+m_{2}\right) l_{1}{ }^{2} \dot{\theta}_{1}{ }^{2}+\frac{1}{2} m_{2} l_{2}{ }^{2} \dot{\theta}_{2}{ }^{2} \\
& +m_{2} l_{1} l_{2} \dot{\theta}_{1} \dot{\theta}_{2}\left(\cos \theta_{1}-\theta_{2}\right) \\
& -\left[m_{1} g l_{1} \sin \theta_{1}+m_{1} g\left(l_{1} \sin \theta_{1}\right.\right. \\
& \left.\left.+l_{2} \sin \theta_{2}\right)\right]
\end{aligned}
$$

$$
\begin{aligned}
=\frac{1}{2} I_{0} \dot{\theta}_{0}+\frac{1}{2}( & \left.m_{1}+m_{2}\right) l_{1}{ }^{2} \dot{\theta}_{1}{ }^{2}+\frac{1}{2} m_{2} l_{2}{ }^{2} \dot{\theta}_{2}{ }^{2} \\
& +m_{2} l_{1} l_{2} \dot{\theta}_{1} \dot{\theta}_{2}\left(\cos \theta_{1}-\theta_{2}\right)-\left(m_{1}\right. \\
& \left.+m_{2}\right) g l_{1} \sin \theta_{1}-m_{2} l_{2} \sin \theta_{2}
\end{aligned}
$$

According to Lagrange equation of motion,

$$
\begin{gathered}
\frac{d}{d t} \frac{\partial L}{\partial \dot{q}}-\frac{\partial L}{\partial q}=\tau \\
\frac{\partial L}{\partial \dot{\theta}_{0}}=I_{0} \dot{\theta}_{0} \\
\frac{d}{d t} \frac{\partial L}{\partial \dot{\theta_{0}}}=I_{0} \ddot{\theta}_{0}
\end{gathered}
$$

$$
\frac{\partial L}{\partial \dot{\theta_{1}}}=\left(m_{1}+m_{2}\right) l_{1}^{2} \dot{\theta}_{1}^{2}+m_{2} l_{1} l_{2} \dot{\theta}_{2}\left(\cos \theta_{1}-\theta_{2}\right)
$$

$$
\frac{d}{d t} \frac{\partial L}{\partial \dot{\theta_{1}}}=\left(m_{1}+m_{2}\right) l_{1}^{2} \ddot{\theta}_{1}+m_{2} l_{1} l_{2} \ddot{\theta}_{2} \cos \left(\theta_{1}-\theta_{2}\right)
$$

$$
-m_{2} l_{1} l_{2} \ddot{\theta}_{2} \sin \left(\theta_{1}-\theta_{2}\right)\left(\dot{\theta}_{1}-\dot{\theta}_{2}\right)
$$

$\frac{\partial L}{\partial \theta_{1}}=-m_{2} l_{1} l_{2} \dot{\theta}_{1} \dot{\theta}_{2} \sin \left(\theta_{1}-\theta_{2}\right)-\left(m_{1}+m_{2}\right) g l_{1} \cos \theta_{1}$

$\therefore \frac{d}{d t} \frac{\partial L}{\partial \dot{\theta}_{1}}-\frac{\partial L}{\partial \theta_{1}}=\tau_{1}=\left(m_{1}+m_{2}\right) l_{1}^{2} \ddot{\theta}_{1}+$ $m_{2} l_{1} l_{2} \ddot{\theta}_{2} \cos \left(\theta_{1}-\theta_{2}\right)-m_{2} l_{1} l_{2} \ddot{\theta}_{2} \sin \left(\theta_{1}-\theta_{2}\right)\left(\dot{\theta}_{1}-\right.$ $\left.\dot{\theta}_{2}\right)-m_{2} l_{1} l_{2} \ddot{\theta}_{2} \sin \left(\theta_{1}-\theta_{2}\right)-\left(m_{1}+m_{2}\right) g l_{1} \cos \theta_{1}$

Eq. 19

$$
\frac{\partial L}{\partial \dot{\theta_{2}}}=m_{2} l_{2}^{2} \dot{\theta}_{2}^{2}+m_{2} l_{1} l_{2} \dot{\theta}_{1} \cos \left(\theta_{1}-\theta_{2}\right)
$$

$$
\begin{aligned}
\frac{d}{d t} \frac{\partial L}{\partial \dot{\theta}_{2}}=m_{2} l_{2}{ }^{2} \dot{\theta}_{2} & +m_{2} l_{1} l_{2} \dot{\theta}_{1} \cos \left(\theta_{1}-\theta_{2}\right) \\
- & m_{2} l_{1} l_{2} \dot{\theta}_{1} \sin \left(\theta_{1}-\theta_{2}\right)\left(\dot{\theta}_{1}-\dot{\theta}_{2}\right)
\end{aligned}
$$

$$
\begin{gathered}
\frac{\partial L}{\partial \theta_{2}}=-m_{2} l_{1} l_{2} \dot{\theta}_{1} \dot{\theta}_{2} \sin \left(\theta_{1}-\theta_{2}\right)-m_{2} g l_{2} \cos \theta_{2} \\
\therefore \frac{d}{d t} \frac{\partial L}{\partial \dot{\theta}_{2}}-\frac{\partial L}{\partial \theta_{2}}=\tau_{2}=m_{2} l_{2}{ }^{2} \ddot{\theta}_{2}+m_{2} l_{1} l_{2} \ddot{\theta}_{1} \cos \left(\theta_{1}-\right. \\
\left.\theta_{2}\right)-m_{2} l_{1} l_{2} \dot{\theta}_{1} \sin \left(\theta_{1}-\theta_{2}\right)\left(\dot{\theta}_{1}-\dot{\theta}_{2}\right)- \\
m_{2} l_{1} l_{2} \dot{\theta}_{1} \dot{\theta}_{2} \sin \left(\theta_{1}-\theta_{2}\right)-m_{2} g l_{2} \cos \theta_{2} \quad \text { Eq. } 20
\end{gathered}
$$

Simplifying Eq. 19 and Eq. 20,

$$
\tau_{0}=I_{0} \ddot{\theta}_{0}
$$

$$
\tau_{1}=\left(m_{1}+m_{2}\right) l_{1}^{2} \ddot{\theta}_{1}+m_{2} l_{1} l_{2} \ddot{\theta}_{2} \cos \left(\theta_{1}-\theta_{2}\right)-
$$


Dynamics of 3 - Links Articulated Robotic Manipulator: A Computational Model

$$
m_{2} l_{1} l_{2} \dot{\theta}_{2}^{2} \sin \left(\theta_{1}-\theta_{2}\right)+\left(m_{1}+m_{2}\right) g l_{1} \cos \theta_{1}
$$

$$
\begin{array}{r}
\tau_{2}=m_{2} l_{2}{ }^{2} \ddot{\theta}_{2}+m_{2} l_{1} l_{2} \ddot{\theta}_{1} \cos \left(\theta_{1}-\theta_{2}\right)- \\
m_{2} l_{1} l_{2} \dot{\theta}_{1}^{2} \sin \left(\theta_{1}-\theta_{2}\right)+m_{2} g l_{2} \cos \theta_{2} \text { Eq. } 23
\end{array}
$$

described by the free body diagram shown in figure 1 .

\section{RESULT ANALYSIS}

The summary of the result of this computation is presented in table I below:

These equations are specific for the three link robotic arm

Table- I: Summary result of the computation

\begin{tabular}{|l|c|l|}
\hline Joints & Torque & Value \\
\hline $\mathrm{J}_{0}$ & $\tau_{0}$ & $I_{0} \ddot{\theta}_{0}$ \\
\hline $\mathrm{J}_{0}$ & $\tau_{1}$ & $\left(m_{1}+m_{2}\right) l_{1}{ }^{2} \ddot{\theta}_{1}+m_{2} l_{1} l_{2} \ddot{\theta}_{2} \cos \left(\theta_{1}-\theta_{2}\right)-m_{2} l_{1} l_{2} \dot{\theta}_{2}{ }^{2} \sin \left(\theta_{1}-\theta_{2}\right)+\left(m_{1}+m_{2}\right) g l_{1} \cos \theta_{1}$ \\
\hline $\mathrm{J}_{2}$ & $\tau_{2}$ & $m_{2} l_{2}{ }^{2} \ddot{\theta}_{2}+m_{2} l_{1} l_{2} \ddot{\theta}_{1} \cos \left(\theta_{1}-\theta_{2}\right)-m_{2} l_{1} l_{2} \dot{\theta}_{1}{ }^{2} \sin \left(\theta_{1}-\theta_{2}\right)+m_{2} g l_{2} \cos \theta_{2}$ \\
\hline
\end{tabular}

In the end of the computation exercise, Table-I shows the dynamics equations at the various joints. These equations represents the values of the torque $(\tau)$ that will be required to cause a displacement at the various joints. Parameter present in these equations are discussed in table-II.

In summary, Table- II shows the parameters produced from the equations

Table-II: Description of parameters present in the equations

\begin{tabular}{|l|c|l|}
\hline Parameter & Symbol & Description \\
\hline Torque & $\tau_{0}, \tau_{1}$ and $\tau_{2}$ & $\begin{array}{l}\text { The value of } \\
\text { forces required to } \\
\text { cause joint } \\
\text { displacement }\end{array}$ \\
\hline Angle & $\theta_{0}, \theta_{1}$ and $\theta_{2}$ & $\begin{array}{l}\text { Value of } \\
\text { displacement on } \\
\text { a joint }\end{array}$ \\
\hline Length of arm & $l_{0}, l_{1}$ and $l_{2}$ & $\begin{array}{l}\text { The value of the } \\
\text { length of the } \\
\text { robot arm at } \\
\text { various joints }\end{array}$ \\
\hline Mass & $m_{0}, m_{1}$ and $m_{2}$ & $\begin{array}{l}\text { The value of the } \\
\text { mass of arm } \\
\text { segment }\end{array}$ \\
\hline
\end{tabular}

Table II shows the descriptions of the parameters used in the computational models shown in table I. The parameters $\tau_{0}, \tau_{1}$ and $\tau_{2}$ represent the force (torque) that must be provided by DC motor to move joints $j_{0}, j_{1}$ and $j_{2}$ to angles specified by $\theta_{0}, \theta_{1}$ and $\theta_{2}$ respectively, while $l_{0}$ to $l_{2}$ are the length of the respective links, and $m_{0}, m_{1}$ and $m_{2}$ are the masses of the various links.

By these computations, the robot easily operates seamlessly with predictable loci while fully operational. The directional motions is resolved with the capability of forward and backward movement without susceptibility to delays and failures in its trajectory.

\section{CONCLUSION}

The process of robotic systems requires the kinematics and dynamics of the robotic manipulators. As a follow up on the computational process of kinematic presented in [10], the computational dynamics of a robotic manipulator has been presented here. A simplified approach involving the use of Lagrange method for the determination of the manipulator dynamics of a 3-links manipulator was considered. In this approach, the energies and forces acting at each joint were computed separately, forming mathematical models for the required torques at those joints.

The results obtained from these analysis show that:

1. The torques at the respective joints in a manipulator is a product of the mass, length, and angle of the joint.

2. The computed values can be used to determine the rating of actuator to be used in the robot implementation.

3. The approach simplified the process of determining the dynamics of joints in a robot manipulator.

4. This is a novel approach to evaluating dynamic 3-links articulated robotic manipulator.

\section{REFERENCES}

1. Richard M., Zcxiang Li, Sastry S., "A Mathematical Introduction to Robotic Manipulation”, CRC Press USA, 1994.

2. Lewis F. L., Dawson D. M. and Abdallah C. T., "Robot Manipulator Control: Theory and Practice", Marcel Dekker, Inc., 270 Madison Avenue, New York, NY 10016, U.S.A., 2004.

3. Featherstone R., "Robot Dynamic", Australian National University, School of Engineering, Canberra, ACT, Australia, 2007. Available at: doi:10.4249/scholarpedia.3829.

4. Asada H. H., "Introduction to Robotics: Chapter 7.", Massachusetts Institute of Technology. Available at: https://ocw.mit.edu/courses/mechanical-engineering/2-12-introductionto-robotics-fall-2005/lecture-notes

5. Craig J. J., "Introduction to Robotics Mechanics and Control", Pearson Prentice Hall, Pearson Education, Inc., Upper Saddle River, NJ 07458, 2005.

6. Jazar R. N., "Theory of Applied Robotics: Kinematics, Dynamics, and Control Second Edition", Springer New York Dordrecht Heidelberg London, 2010.

7. Featherstone $\mathrm{R}$ and Orin D., "Robot Dynamics: Equations and algorithms", Proc. IEEE Int. Conf. Robotics \& Automation, San Francisco, CA, pp. 826 - 834, 2000.

8. Ben-Ari M. and Mondada F., "Elements of Robotics", Springer Open, 2018. Available at https://doi.org/10.1007/978-3-319-62533-1.

9. Maplesoft, "Robot Manipulators Position, Orientation and Coordinate Transformations", Available at https://www.maplesoft.com/content/EngineeringFundamentals/13/Map leDocument_13.

Published By: Blue Eyes Intelligence Engineering 
10. Obasi C. C., Ikharo A. B., Balogun V. A., Udaba A., Ogbewey L. I, "Computational Analysis of Kinematics of 3 - Links Articulated Robotic Manipulator", International Journal of Engineering and Advanced Technology, Vol. 9, No. 2, 2019. Available at: DOI: 10.35940/ijeat.F8631.129219.

\section{AUTHORS PROFILE}

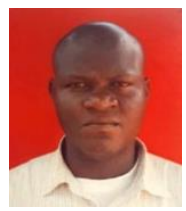

Chukwuemeka C. Obasi, received B.Eng degree in Computer Engineering from Enugu State University of Science and Technology, Enugu, Nigeria, M.Eng in Electronics and Computer Engineering with specialization in Computer \& Control Engineering from Nnamdi Azikiwe University, Awka, Nigeria. He is currently a Ph.D student at the University of Nigeria, Nsukka, Nigeria. $\mathrm{He}$ is a lecturer in the department of Computer Engineering, Edo University Iyamho, Edo State, Nigeria. He presently has research interest in Embedded Systems, Internet of Things (IoT), Biosensors, Cloud Computing, Machine Learning and Robotics.

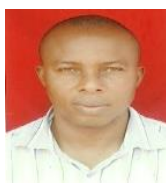

Ikharo A. Braimoh, received his B.Eng in Electrical/Electronics Engineering, M.Eng in Electronics Engineering and Ph.D in Communications Engineering from Abubakar Tafawa Balewa University, Bauchi. He is a Lecturer in the Department of Computer Engineering, Edo University Iyamho. His research interests include Mobile Cellular and Wireless Communication, Computer Networks and Data Communication, Big Data Analytics and Internet of Things, Modeling of Tropospheric Effects on Radio Wave Propagation for Communications.

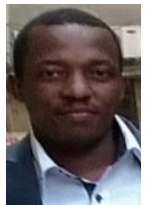

Alpheous Udaba, is a lecturer at the department of Electrical/Electronics, Air Force Institute of Technology, Kaduna, Nigeria. His research interests are in embedded systems, energy harvesting and robotics.

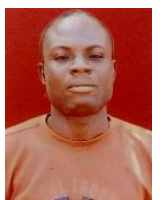

Leonard Iyase Ogbewey, obtained first and second degrees in Computer Engineering. He is a lecturer in the Department of Computer Engineering, Federal Polytechnic Offa Kwara State, Nigeria. He has been researching on different arms of computer engineering, including embedded systems, Algorithm development, and neural networks.

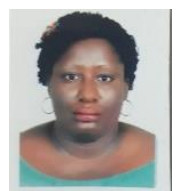

Bambe Adeduntan Oluyomi is currently pursuing a master degree in Computer Engineering at the Ladoke Akintola University of Technology, Ogbomoso, Nigeria Her research interest include embedded system and neural networks 